\title{
An Advanced Susceptible-Exposed-Infectious-Recovered model for quantitative analysis of COVID-19
}

\author{
RAHUL DIXIT $^{1, *(D)}$, DEV SOURAV PANDA ${ }^{2}$ and SHRADHA SUMAN PANDA ${ }^{3}$ \\ ${ }^{1}$ Department of Computer Science and Engineering, Indian Institute of Information Technology Pune, Pune, \\ Maharashtra 411 048, India \\ ${ }^{2}$ Department of Electronics and Communication Engineering, Indian Institute of Information Technology Pune, \\ Pune, Maharashtra 411 048, India \\ ${ }^{3}$ Department of Medical Technology, Indian Institute of Technology Jodhpur-All India Institute of Medical \\ Science Jodhpur (Joint Program), Jodhpur, Rajasthan 342 037, India \\ e-mail: rahul2012ism@gmail.com
}

MS received 10 August 2020; revised 7 February 2021; accepted 21 February 2021

\begin{abstract}
The infectious diseases with a viral origin are of significant worldwide concern. In recent times, pandemics are creating havoc across the entire globe. This paper presents a constructive analysis of a new mathematical concept that will help the medical authorities to predict and to take controlling measures. In this work, we use ordinary first-order differential equations and compartmental model analysis for calculation of infection rate, transmission rate, and reproduction number of the patients. A new Advanced Susceptible-Exposed-Infectious-Recovered model has been introduced, which has greater accuracy of the reproduction number. The prediction of mode of disease transmission demonstrates the performance characteristics of the proposed model.
\end{abstract}

Keywords. COVID-19; coronavirus; Advanced Susceptible-Exposed-Infective-Recovered model; environmental compartment.

\section{Introduction}

COVID-19 (CoronaVirus Disease 2019) is caused by a new strain of coronaviruses having viral origin. It is spreading rapidly across the world and has been declared a pandemic. Coronaviruses are a family of viruses that have spikes protruding from their surface and are contagious in nature. These viruses contain essential protein packages surrounding the genetic material. According to a survey report the first human coronavirus $(\mathrm{HCoV})$ was isolated in the year 1937 [1] but, later in the year 1965, the virus got its name as coronavirus due to its "crown"-like appearance when seen under an electronic microscope.

On $31^{\text {st }}$ December 2019 a new strain of coronavirus (n-CoV) was first identified as the cause of COVID-19 outbreak that emerged in the city of Wuhan, Hubei provinces, China, thereby making Wuhan as the original epicentre of this novel coronavirus outbreak. SARS-CoV 2 (Severe Acute Respiratory Syndrome-CoronaVirus 2) proteins are the viruses responsible for COVID-19, the nomenclature being done by World Health Organization (WHO) on 11th February 2020. There have been remarkable progresses on SARS-CoV 2 treatment; however

*For correspondence

Published online: 28 April 2021 complete eradication of COVID-19 is still out of reach, though many solidarity trials of different drugs are being practiced. Introduction of a new mathematical model can be considered as one of the ways to fit the need of the hour. In the absence of a successful vaccine, mathematical modeloriented interventions can be considered as an alternative strategy for reducing the infection burden.

In recent years, several mathematical models have been proposed for infectious diseases [2-6]. These models helped the medical authorities in predicting the dynamics and behavior of infectious diseases. In [2] the authors have computed the crucial epidemiological model parameters for prediction of the spread of SARS-CoV 2 outbreak. It was concluded that imposing social distancing can help minimize the spread of this pandemic. In [3] authors have presented a compartmental mathematical model but have not used environmental compartment as proposed in this paper. This compartment will significantly affect the cause for this pandemic. In [5] authors have used four coupled non-linear ordinary differential equations (ODEs) for detection of leukaemia/lymphoma virus. They compute sensitivity indices of the reproductive numbers, which measure the initial disease transmission. However, their model used four compartments only. Wu et al [7] suggested a SEIR (Susceptible-Exposed-Infectious-Recovered) model 
for COVID-19. In this model authors describe the transmission dynamics as well as reproduction number of the disease, which comes out to be 2.68. Tang et al [8] analyzed an individual-level epidemiological status and disease control strategies for COVID-19. Read et al [9] proposed their model based on the data fitting of simple SEIR components. According to this model, authors assume Poisson-distributed daily time increments and report the reproduction number of the disease as 3.1. Zhu et al [10] proposed another model with an additional concept of deep learning algorithms. In this model, the authors analyzed the potential hosts and the impact of the deadly SARS-CoV 2. The estimated outputs of this model deviate from the originally recorded data, which is the major drawback of this model. In $[35,36]$ the authors simulate and predict the model, thereby concluding the oscillatory dynamics of COVID-19 in future. The paper also states the strategies to control the long-term impact of the disease. According to the state-of-the-art of COVID-19 mathematical models, it is observed that the calculation of disease transmission number and reproduction number does not match the mathematical model and actual data because the environmental component is not used in the model. In our present model we propose an additional new compartment for the coronavirus concentration in the environmental reservoir, which gives a greater accuracy of the reproduction number and prediction of the mode of disease transmission.

\subsection{Our motivation and contributions}

In this paper, we propose a mathematical model that is capable of calculating reproduction number and is able to predict the mode of transmission of COVID-19. According to state-of-the art of COVID-19 [11-15] authors have mainly used ordinary first-order differential equations and compartmental model analysis (CMA) for calculation of transmission rate and reproduction number, but such methods suffer from low accuracy. In this work we propose an Advanced Susceptible-Exposed-Infectious-Recovered (ASEIR) model in which we introduce additional compartments, namely quarantined $(Q)$, diagnosed $(J)$, and the environmental reservoir $(V)$. The inclusion of the environmental compartment into the model affects the reproduction number to a great extent, thereby increasing the accuracy of the quantification.

In order to formulate the control strategies, our major contributions are as follows:

a) Knowing the severity of the pandemic by calculating basic reproduction number.

b) Prediction of the graph of the infectious patients and its comparison to the reported graph.

c) Prediction of the mode of transmission of the disease.

The remaining paper is arranged as follows. In the next section, we present assumptions and problem statement of our proposed model. The proposed ASEIR model is discussed in section 3. Section 4 presents numerical simulations. Finally, the conclusions with future research directions are provided in section 5.

\section{Mathematical formulations}

The intensity of an infectious disease outbreak can be predicted with the help of basic reproduction number $\left(R_{0}\right)$ as mentioned earlier. For the characterization of a disease as pandemic or for any large publicized outbreaks, this concept is essential. $R_{0}$ estimations have been an important part during the 2003 SARS pandemic [16] and 2012 MERS [17] outbreak along with 2014 Ebola epidemic [18] in West Africa. The category of disease depends on value of $R_{0}$ as $[19,20]$ follows:

$$
\text { disease }=\left\{\begin{array}{c}
\text { normal if } R_{0} \leq 1 \\
\text { epidemic if } 1<R_{0} \leq 1.5 \\
\text { pandemic otherwise }
\end{array}\right.
$$

\subsection{Definitions and assumptions}

Many scientists and epidemiologists are still busy in finding the vaccines and drugs for treatment of the current pandemic disease. Therefore, it is necessary for the mathematicians to formulate certain estimations and control strategies for the same. Mathematical modeling is one way of analyzing the pandemic through various pandemiological characteristics and the basic reproduction number.

2.1a Definitions: According to the available data of COVID-19, we categorize the people into seven compartments, as follows:

- Susceptible. This compartmental characteristic includes people who are healthy but vulnerable to the disease. These people can get infection when they come in close contact with the exposed and infected patients. They can also be infected through animal-tohuman transmission. In our proposed model, it is denoted by $S(t)$.

- Exposed. This compartmental characteristic includes people who are under the incubation period of about 14 days, after being affected by SARS-CoV 2. During this time, they are capable of infecting others (possibly with very low infectivity). They are asymptomatic and gradually show symptoms after the incubation period. In our proposed model, it is denoted by $E(t)$.

- Infectious. This compartmental characteristic includes people who got the infection either through the direct contact with other infected patients or through wildlife transmission. They show strong infectivity and are symptomatic. Yet they are not quarantined. Here, it is denoted by $I(t)$.

- Quarantined. It includes that fraction of population who have been infected by the disease, but are 
asymptomatic. They are kept isolated and are not yet diagnosed by the medical authorities. In the proposed model it is represented by $Q(t)$.

- Diagnosed. This compartmental characteristic includes people who have been infected, and are under proper medical supervision. During this stage, some of the patients die and some recover. In the proposed work, it is denoted as $J(t)$.

- Recovered. This compartment describes people who recover from the disease and now fall into the susceptible compartment again. There may be chances that one person can be attacked by this disease for more than once. In our proposed model, it is represented by $R(t)$.

- Environment. It is an additional proposed compartment that we have introduced in our work, in order to quantify the severity of the disease more precisely. In this compartment, the exposed and infected individuals are mainly responsible for the spread of coronavirus in the atmosphere. Then either through direct contact or through animal-to-human transmission, these viruses spread into the susceptible compartment's people. In our work, we have denoted this compartment as $V(t)$.

2.1b Assumptions: In order to reduce the complexity of the equations and to analyze the situation more clearly we assume following:

- In a country or a territory, the distribution of population is homogenous.

- The total population in a country remains constant. The numbers of new births and deaths are not taken into consideration during the pandemic. This means deaths are only due to the disease.

- Climatic interference is rejected since the spread of COVID-19 due to climatic change is not mentioned anwhere.

- All the direct contacts by the exposed and infected patients with the susceptible ones (neglecting contact with other compartmental people) are capable of transmitting infection.

A schematic diagram representing the flow of people in various compartmental parameters is given in figure 1 .

\section{The proposed ASEIR model}

In this section, we present the proposed ASEIR model in detail. Initially, we discuss the initial conditions taken for calculation. Further, we have used ODEs and calculated the infection and transition matrix. Finally, the calculation of reproduction number predicts the severity of disease. These steps are detailed in the following subsections.

\subsection{Initial conditions}

The initial conditions used in this work are as follows:

(a) Parameters $\beta_{1}(t), \beta_{2}(t)$, and $\beta_{3}(t)$ are all positive, non-increasing functions such that the reduction of transmission rates is well determined by the higher values of $E, I$, and $V$ components. We have considered two cases:

(1) $\beta_{1}(t)>0, \beta_{2}(t)>0, \beta_{3}(t)>0$

(2) $\beta \prime_{1}(t) \leq 0, \beta \prime_{2}(t) \leq 0$, and $\beta \prime_{3}(t) \leq 0$ where $\beta \prime(t)$ denotes the derivative of the function $\beta(t)$.

(b) At time $t=0$ susceptible parameter is denoted by $S(0)$, which is equal to $\frac{\lambda}{\mu}$, and rest of the compartmental parameters are equated to 0 .

\subsection{Calculation of infection and transition matrix}

We describe the transmission dynamics of the COVID19 outbreak, using the following differential equations:

$$
\left.\begin{array}{r}
\text { susceptible rate }\left(\frac{d S}{d t}\right)=\eta-\beta_{1} S E-\beta_{2} S I-\beta_{3} S V-\mu S \\
\text { exposed rate }\left(\frac{d E}{d t}\right)=\beta_{1} S E+\beta_{2} S I+\beta_{3} S V-\left(\frac{\varepsilon+\lambda}{\alpha}+\mu\right) E \\
\text { infectious rate }\left(\frac{d I}{d t}\right)=\frac{\lambda}{\alpha} E-\left(\mu_{1}+\frac{\delta}{\alpha}+\mu\right) I \\
\text { quarantined rate }\left(\frac{d Q}{d t}\right)=\frac{\varepsilon}{\alpha} E-\left(\frac{\theta}{\alpha}+\mu\right) Q \\
\text { diagnosed rate }\left(\frac{d J}{d t}\right)=\frac{\delta}{\alpha} I+\frac{\theta}{\alpha} Q-\left(\gamma+\mu_{2}\right) J \\
\text { recovery rate }\left(\frac{d R}{d t}\right)=\gamma J-\mu R \\
\text { environmental rate }\left(\frac{d V}{d t}\right)=\xi_{1} E+\xi_{2} I-\sigma V
\end{array}\right\}
$$

where all the model parameters are defined in table 1 . In this system of equations, the term $\alpha$ represents the incubation period between the infection in an individual and the onset of symptoms. It is important to note that we always divide $\alpha$ with the fractions of people who are either infected or exposed to the viruses. This is done to get the daily information of affected individuals.

In this set of equations, the compartmental parameters producing infection are $E, I$, and $V$ only since people under other compartments are either vulnerable to infection (but not infected) or under proper guidance of medical authorities. Hence with the help of the infection 


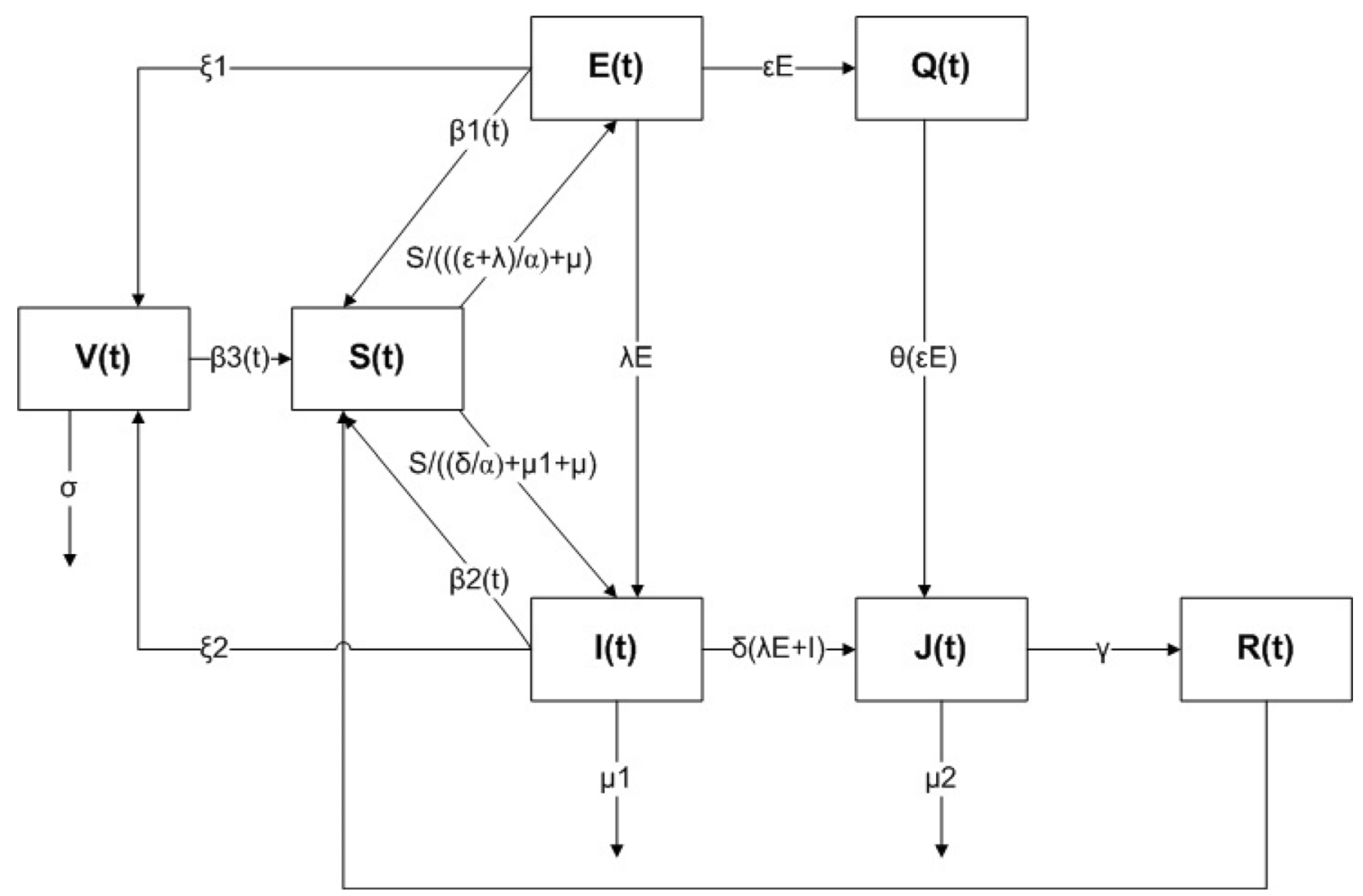

Figure 1. The distribution of Indian population in various compartments of ASEIR model.

Table 1. Biologically relevant parameters of India: definitions and values.

\begin{tabular}{|c|c|c|c|c|}
\hline S1 no. & Model parameters & Biological definitions & Estimated parameter values & Sources \\
\hline 1. & $\eta$ & Influx rate of the population. & 3436.69 per day & {$[27]$} \\
\hline 2. & $\sigma$ & Rate of removal of virus from the environmental reservoir. & 1 per day & [23] \\
\hline 3. & $\beta_{1}(0)$ & Transmission constant between susceptible and exposed. & $1.86 \times 10^{-8}$ per person per day & [24] \\
\hline 4. & $\beta_{2}(0)$ & Transmission constant between susceptible and infected. & $0.32 \times 10^{-8}$ per person per day & {$[28]$} \\
\hline 5. & $\beta_{3}(0)$ & Transmission constant between susceptible and environment. & Fit to data & - \\
\hline 6. & $\mu$ & Natural death rate. & $4.88 \times 10^{-3}$ per day $=7.309 \%$ & {$[21]$} \\
\hline 7. & $\mu_{1}$ & Death rate of infected patient. & $1.86 \times 10^{-4}$ per day $=2.877 \%$ & {$[22]$} \\
\hline 8. & $\mu_{2}$ & Death rate of diagnosed patient. & $2.89 \times 10^{-4}$ per day $=4.477 \%$ & [29] \\
\hline 9. & $\alpha$ & Incubation period between infection and onset of symptoms. & $2-14$ days $=7$ days $($ mean $)$ & {$[23]$} \\
\hline 10. & $\gamma$ & Rate of recovery of diagnosed patients. & 1 out of 250 per day $=61.98 \%$ & [23] \\
\hline 11. & $\xi_{1}$ & Rate of shedding of viruses by exposed. & Fit to data & - \\
\hline 12. & $\xi_{2}$ & Rate of shedding of viruses by infected. & $10^{-9}$ per person per day per $\mathrm{ml}$ & {$[36]$} \\
\hline 13. & $\varepsilon$ & Transmission rate from exposed to quarantined compartment. & 0.42 & - \\
\hline 14. & $\lambda$ & Transmission rate from exposed to infected compartment. & 0.51 & - \\
\hline 15. & $\theta$ & Transmission rate from quarantined to diagnosed compartment. & 0.60 & - \\
\hline 16. & $\delta$ & Transmission rate from infected to diagnosed compartment. & 0.90 & - \\
\hline 17. & $v$ & Positive adjusting coefficient of the transmission rates. & Fit to data & - \\
\hline
\end{tabular}

components, i.e. $E, I$, and $V$, we can find the infection matrix $(F)$ and the transition matrix $(T)$ of the COVID19 disease as

$$
(F)=\left[\begin{array}{ccc}
\beta_{1}(0) S_{0} & \beta_{2}(0) S_{0} & \beta_{3}(0) S_{0} \\
0 & 0 & 0 \\
0 & 0 & 0
\end{array}\right]
$$




$$
(T)=\left[\begin{array}{ccc}
\frac{\varepsilon+\lambda}{\alpha}+\mu & 0 & 0 \\
-\lambda & \mu_{1}+\frac{\delta}{\alpha}+\mu & 0 \\
-\xi_{1} & -\xi_{2} & \sigma
\end{array}\right] .
$$

Next, the severity of the disease is described by calculating the reproduction number. This number will determine the devastating impact of the disease on a country or a territory.

\subsection{Calculation of basic reproduction number}

In a country or a territory, the severity of any disease is known by its reproduction number. Basically, the term "reproduction number" defines the total secondary infections caused by a single infected patient. Mathematically it refers to spectral radius of next generation matrix, formed by matrix multiplication of infection matrix and inverse of transition matrix $\left(F T^{-1}\right)$ [12].

Initially, we begin with calculation of inverse transition matrix. In order to find the inverse, we need to first calculate its augmented matrix as

$$
\operatorname{aug}(T)=\left[\begin{array}{ccc|ccc}
\mid \frac{\varepsilon+\lambda}{\alpha}+\mu & 0 & 0 & 1 & 0 & 0 \\
-\lambda & \mu_{1}+\frac{\delta}{\alpha}+\mu & 0 & 0 & 1 & 0 \\
-\xi_{1} & -\xi_{2} & \sigma & 0 & 0 & 1
\end{array}\right] .
$$

Now we need to make the left side of the augmented matrix as the identity matrix and the right side will be required for inverse transition matrix.

$$
\begin{aligned}
& \operatorname{aug}(T)=\left[\begin{array}{ccc|ccc}
1 & 0 & 0 & \frac{1}{\varepsilon+\lambda}+\mu & 0 & 0 \\
0 & \mu_{1}+\frac{\delta}{\alpha}+\mu & 0 & \frac{\lambda}{\varepsilon+\lambda} & 1 & 0 \\
0 & -\xi_{2} & \sigma & \frac{\xi_{1}}{\varepsilon}+\mu & 0 & 1
\end{array}\right] \\
& \Rightarrow \operatorname{aug}(T)=\left[\begin{array}{ccc|cc}
\frac{\frac{1}{\varepsilon+\lambda}+\mu}{\alpha}+\mu & 0 & 0 \\
\left|\begin{array}{ccc}
1 & 0 & 0 \\
0 & 1 & 0 \\
0 & -\xi_{2} & \sigma
\end{array}\right| \frac{1}{\left(\frac{\varepsilon+\lambda}{\alpha}+\mu\right)\left(\mu_{1}+\frac{\delta}{\alpha}+\mu\right)} \frac{1}{\mu_{1}+\frac{\delta}{\alpha}+\mu} & 0 \\
\frac{\frac{\xi_{1}}{\varepsilon+\lambda}}{\alpha}+\mu & 0 & 1
\end{array}\right]
\end{aligned}
$$

$$
\Rightarrow \operatorname{aug}(T)=\left[\begin{array}{ccccc} 
& \frac{1}{\frac{\varepsilon+\lambda}{\alpha}+\mu} & 0 & 0 \\
& \frac{1}{\lambda} & 0 \\
\left|\begin{array}{lll}
1 & 0 & 0 \\
0 & 1 & 0 \\
0 & 0 & 1
\end{array}\right| & \frac{\xi_{1}\left(\mu_{1}+\frac{\delta}{\alpha}+\mu\right)+\lambda \xi_{2}}{\alpha}+\mu & \\
& & \frac{\xi_{2}}{\sigma\left(\frac{\varepsilon+\lambda}{\alpha}+\mu\right)\left(\mu_{1}+\frac{\delta}{\alpha}+\mu\right)} & \frac{\xi_{2}\left(\mu_{1}+\frac{\delta}{\alpha}+\mu\right)}{\sigma}
\end{array}\right] .
$$

Therefore, the inverse of the transition matrix is the right side of the augmented matrix with left side as the identity matrix:

$$
T^{-1}=\left[\begin{array}{ccc}
\frac{1}{\frac{\varepsilon+\lambda}{\alpha}+\mu} & 0 & 0 \\
\frac{\lambda}{\left(\frac{\varepsilon+\lambda}{\alpha}+\mu\right)\left(\mu_{1}+\frac{\delta}{\alpha}+\mu\right)} & \frac{1}{\mu_{1}+\frac{\delta}{\alpha}+\mu} & 0 \\
\frac{\xi_{1}\left(\mu_{1}+\frac{\delta}{\alpha}+\mu\right)+\lambda \xi_{2}}{\sigma\left(\frac{\varepsilon+\lambda}{\alpha}+\mu\right)\left(\mu_{1}+\frac{\delta}{\alpha}+\mu\right)} & \frac{\xi_{2}}{\sigma\left(\mu_{1}+\frac{\delta}{\alpha}+\mu\right)} & \frac{1}{\sigma}
\end{array}\right] .
$$

We know that the reproduction number $R_{0}$ is the spectral radius of the next generation matrix. This implies $R_{0}=\rho\left(F T^{-1}\right)$, where $\rho$ is spectral operator.

$$
\begin{gathered}
\Rightarrow R_{0}=\left[\begin{array}{ccc}
\beta_{1}(0) S_{0} & \beta_{2}(0) S_{0} & \beta_{3}(0) S_{0} \\
0 & 0 & 0 \\
0 & 0 & 0
\end{array}\right] \\
{\left[\begin{array}{ccc}
\frac{1}{\frac{\varepsilon+\lambda}{\alpha}+\mu} & 0 & 0 \\
\frac{\lambda}{\left(\frac{\varepsilon+\lambda}{\alpha}+\mu\right)\left(\mu_{1}+\frac{\delta}{\alpha}+\mu\right)} & \frac{1}{\mu_{1}+\frac{\delta}{\alpha}+\mu} & 0 \\
\frac{\xi_{1}\left(\mu_{1}+\frac{\delta}{\alpha}+\mu\right)+\lambda \xi_{2}}{\sigma\left(\frac{\varepsilon+\lambda}{\alpha}+\mu\right)\left(\mu_{1}+\frac{\delta}{\alpha}+\mu\right)} & \frac{\xi_{2}}{\sigma\left(\mu_{1}+\frac{\delta}{\alpha}+\mu\right)} & \frac{1}{\sigma}
\end{array}\right]} \\
\Rightarrow R_{0}=\frac{\beta_{1}(0) S_{0}}{\frac{\varepsilon+\lambda}{\alpha}+\mu}+\frac{\beta_{2}(0) S_{0} \lambda}{\left(\frac{\varepsilon+\lambda}{\alpha}+\mu\right)\left(\mu_{1}+\frac{\delta}{\alpha}+\mu\right)}+\frac{\beta_{3}(0) S_{0}\left[\xi_{1}\left(\mu_{1}+\frac{\delta}{\alpha}+\mu\right)+\lambda \xi_{2}\right]}{\sigma\left(\frac{\varepsilon+\lambda}{\alpha}+\mu\right)\left(\mu_{1}+\frac{\delta}{\alpha}+\mu\right)} \\
\Rightarrow R_{0}=R_{1}+R_{2}+R_{3} .
\end{gathered}
$$

Comparing Eqs. (4.3) and (4.4), we get

$$
\begin{gathered}
\Rightarrow R_{1}=\frac{\beta_{1}(0) S_{0}}{\frac{\varepsilon+\lambda}{\alpha}+\mu} \\
\Rightarrow R_{2}=\frac{\beta_{2}(0) S_{0} \lambda}{\left(\frac{\varepsilon+\lambda}{\alpha}+\mu\right)\left(\mu_{1}+\frac{\delta}{\alpha}+\mu\right)}
\end{gathered}
$$




$$
\Rightarrow R_{3}=\frac{\beta_{3}(0) S_{0}\left[\xi_{1}\left(\mu_{1}+\frac{\delta}{\alpha}+\mu\right)+\lambda \xi_{2}\right]}{\sigma\left(\frac{\varepsilon+\lambda}{\alpha}+\mu\right)\left(\mu_{1}+\frac{\delta}{\alpha}+\mu\right)} .
$$

These reproduction numbers quantify the severity level of an epidemic. Here, $R_{1}$ denotes the impact of the disease due to the transmission of coronavirus from exposed to susceptible individuals; $R_{2}$ denotes the measure of severity of COVID-19 on the susceptible due to the infected individuals; and $R_{3}$ measures the contribution of the environment on the susceptible. The summation of all the three reproduction numbers collectively determines the severe impact of COVID-19 on a country or a territory.

\section{Numerical simulations}

For the purpose of numerical simulations, we applied our proposed mathematical model on Indian database. We have taken the daily data from WHO [30], ICMR [34], and other official resources in order to verify our model. For the sake of numerical simulations, we need to assume certain functions for the transmission rates mentioned in our model. We all know that according to the stability analysis the severity quantification and the transmission rates must be positive and non-decreasing and hence our assumed functions should be non-decreasing.

Let us assume the following functions:

$$
\left.\begin{array}{l}
\Rightarrow \beta_{1}(E)=\frac{\beta_{1(0)}}{1+v E} \\
\Rightarrow \beta_{2}(I)=\frac{\beta_{2(0)}}{1+v I} \\
\Rightarrow \beta_{3}(V)=\frac{\beta_{3(0)}}{1+v V}
\end{array}\right\}
$$

where $\beta_{1}(0), \beta_{2}(0)$, and $\beta_{3}(0)$ are positive constants representing the optimum values of the transmission rates, and $v$ is a positive coefficient used for adjusting the values of the transmission rates.

\subsection{Proposed model calibration}

For India we simulated our model for the epidemic period starting from the first COVID-19 positive case (on 30 ${ }^{\text {th }}$ January 2020) in Kerala to 2nd July 2020, i.e. over a period of 155 days. We used the data from various government resources of the country. As per the government report, during these 155 days, 532687 migrants were present in India [18, 25-27]. According to research works coronaviruses can stay active in the environment for a few hours to several days [23], and we assume that the rate of removal of virus from the environment $(\sigma)$ is 1 per day. The transmission constants from exposed and infected patients are taken from a recent study $[15,28]$. The natural death rate and the death rates of the infection-induced and diagnosed patients are taken from the WHO official link [23, 32]. Generally it is analyzed that the incubation period of the disease is from 2 days to 14 days and hence we have taken its mean value, i.e. 7 days for the simulation. On an average 1 out of 250 confirmed patients of COVID-19 recovers per day, implying that the recovery rate of a patient is $\gamma=\frac{1}{250}$ per day [23, 33]. In India, the government is working sincerely and is more active in isolation of the infected patients. Also due to their proper diagnosis, the probability of spreading the coronaviruses into the environment by an infected individual is very less. Therefore, we have considered the virus shedding rate from an infected individual $\left(\xi_{2}\right)$ to be negligible [36]. Still the viruses get into the environmental reservoir from the exposed individuals, in spite of creating awareness by the government and many private agencies. All the model parameters along with the transfer rate constants between the compartments are shown in a tabular form in table 1 . In other models the environmental compartment is not taken into consideration, which shows a large impact on the susceptible members.

\subsection{Initial conditions and estimation of the model parameters}

In the proposed method now we have three model parameters, $\beta_{3}(0), \xi_{1}$, and $v$, that are yet to be determined. For their estimation let us fit the model to the reported data of India [23] from the first corona positive case found in Kerala, i.e. on 30th January 2020, to 2nd July 2020 using the polyfit function in Non-Linear Least Squares method. According to the reported data as on 30th January, we are given the initial conditions in table 2 . Here the initial susceptible population is considered as the net population of the country, including the migrants $[18,25,26]$.

The unknown parametric values can now be estimated using the initial condition, data, and the assumed function in Eq. (5). We have tabulated the parameters and their 95\% confidence intervals in table 3. The Normalized Mean Square Error (NMSE) for the data fitting is simulated as 0.0006 .

Table 2. Initial conditions of the model parameters.

\begin{tabular}{ll}
\hline Model parameters & Initial values \\
\hline$S(0)$ & 13586631 \\
$E(0)$ & 0 \\
$I(0)$ & 1 \\
$Q(0)$ & 0 \\
$J(0)$ & 0 \\
$R(0)$ & 0 \\
\hline
\end{tabular}


Table 3. Estimation of model parameters by Non-Linear Least Squares method.

\begin{tabular}{lll}
\hline Unknown parameters & \multicolumn{1}{c}{ Fitting value } & $95 \%$ confidence intervals \\
\hline$\beta_{3}(0)$ & $0.78 \times 10^{-8}$ & $\left(0,4.067 \times 10^{-8}\right)$ \\
$\xi_{1}$ & 1.93 & $(0,19.003)$ \\
$v$ & $1.07 \times 10^{-4}$ & $\left(0.668 \times 10^{-4}, 1.169 \times 10^{-4}\right)$ \\
\hline
\end{tabular}

\subsection{Basic reproduction number calculation}

We have estimated the unknown model parameters so far. Now, based on the fitting values of these parameters, let us calculate the individual reproduction numbers $R_{1}, R_{2}$, and $R_{3}$ and sum them up to know the average reproduction number of India. Using Eq. (4.5), we get the value of $R_{1}$ as 1.51. Similarly, using Eqs. (4.6) and (4.7), we obtain $R_{2}$ and $R_{3}$ as 0.84 and 1.22 , respectively. Therefore, according to Eq. (4.4), the basic reproduction number $\left(R_{0}\right)$ for the pandemic sums up to 3.57. As discussed, the value of $R_{0}$ quantifies the severity of any epidemic. Since the value of $R_{0}$ is 3.57 , this shows that the disease still has severe impact mainly on the susceptible individuals [29]. Also, we have individually evaluated the values of $R$ due to transmission of coronavirus from exposed-tosusceptible, infected-to-susceptible, and from the environment. Note that highest infections spread from the exposed individuals because they are asymptomatic, and alternatively the infected patients spread least infections to the susceptible, thereby validating our model. Also, one should not miss the significant contribution of the environment in spreading the infection. This proves that environment can be a major mode for the transmission of the disease.

\subsection{Reported vs. predicted curves of infectious patients}

According to the reported data of WHO, the total number of infectious cases and the death tolls are raising exponentially all across the globe. US is the worst hit of this disease, crossing one lakh death cases within a few months. In this aspect, India has controlled the situation to a great extent. However, still the rate of infectious growth is higher compared with the recovery rate. Figure 2 shows the reported cumulative confirmed cases of COVID-19 in India till 2nd July 2020 [30, 31]. From the curve, we can see that there is an exponential rise in the number of confirmed cases of the disease during these 155 days.

Now with the help of this curve and the functions that we have defined in equation (5), we have predicted a graph for a total of 400 days using MATLAB and compared the same

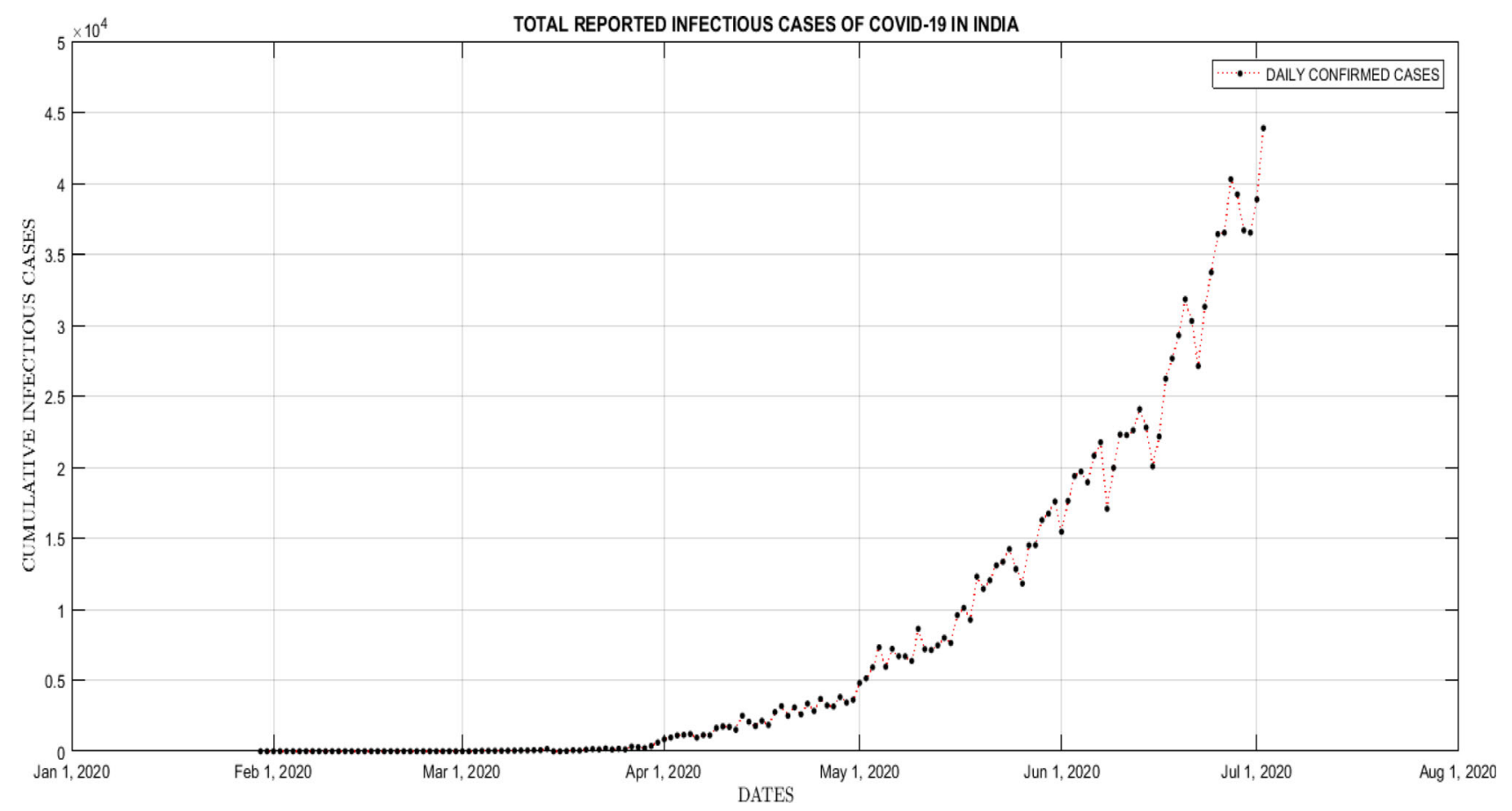

Figure 2. Cumulative infectious cases of COVID-19 in India between 30th January 2020 and 2nd July 2020. 


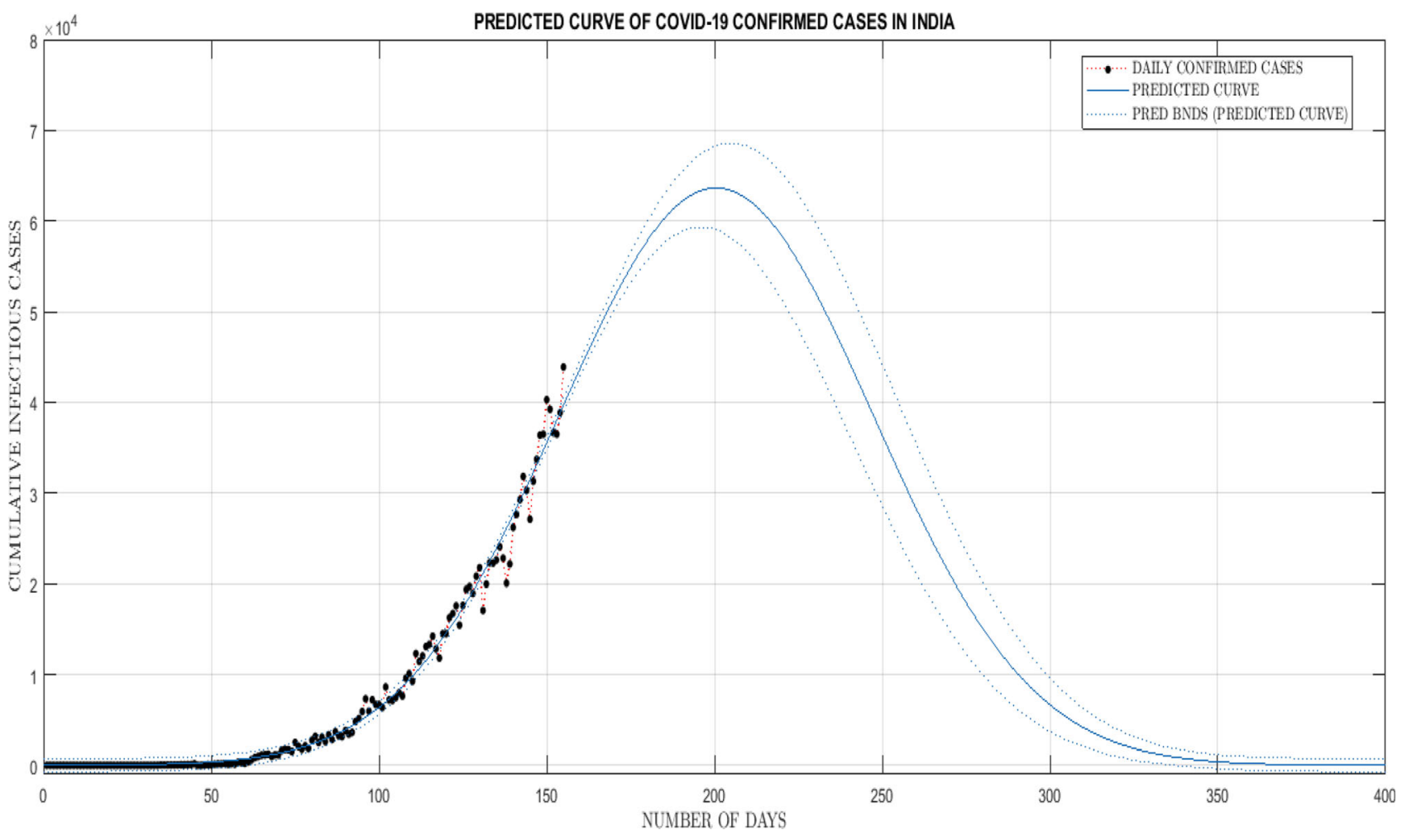

Figure 3. The actual plot (in dots) vs. the predicted plot (in line) of the cumulative infectious cases of COVID-19 in India.

Table 4. Performance comparison with state-of-the-art.

\begin{tabular}{llcc}
\hline Paper & $\begin{array}{c}\text { Mathematical } \\
\text { model }\end{array}$ & $\begin{array}{c}\text { RMSE } \\
\text { value }\end{array}$ & $\begin{array}{c}\text { NMSE } \\
\text { value }\end{array}$ \\
\hline $\begin{array}{l}\text { Sarkar } \text { et al [2] } \\
\text { Khajanchi and Sarkar }\end{array}$ & SARII $\mathrm{S}_{\mathrm{q}}$ & 187.465 & 0.0391 \\
[36] & SAIUQR & 29.984 & 0.0084 \\
Proposed model & ASEIR & 15.289 & 0.0006 \\
\hline
\end{tabular}

to the recorded curve. Figure 3 shows the comparison of our predicted curve and the reported curve.

\subsection{Comparison with state-of-the-art}

In this section, we compare the performance of the proposed mathematical model to those of other mathematical models on the basis of Root Mean Square Error (RMSE) and Normalized Mean Square Error (NMSE).

RMSE is defined as the measure of accuracy of the fitting data. It is given by

$$
\mathrm{RMSE}=\sqrt{\frac{\sum_{i=1}^{n}(O(i)-P(i))^{2}}{n} .}
$$

The NMSE of the model output prediction is defined as

$$
\mathrm{NMSE}=\frac{\sum_{i=1}^{n}(O(i)-P(i))^{2}}{\left(\sum_{i=1}^{n} O(i)\right)\left(\sum_{i=1}^{n} P(i)\right)}
$$

where $n$ indicates the overall size of the observed data, $O(i)$ represents the daily confirmed COVID-19 cases, and $P(i)$ defines the predicted data. Values of the NMSE that are close to 0 denote better model performance. NMSE is a measure of the spread between observed and predicted quantities.

The state-of-the-art models used for comparison in this paper are as follows:

- $\operatorname{SARII}_{\mathrm{q}} \mathrm{S}_{\mathrm{q}}$ - Mathematical model proposed by Sarkar et al [2],

- SAIUQR -Mathematical model proposed by Khajanchi and Sarkar [36].

The performance of the proposed mathematical model is compared in terms of RMSE values and NMSE values, in table 4 and figure 4, respectively. From table 4 and figure 4 it is evident that the proposed model achieves lower RMSE value as well as NMSE value in comparison with other existing models, i.e. 15.289 and 0.0006, respectively. Therefore, we can say that the proposed ASEIR model 

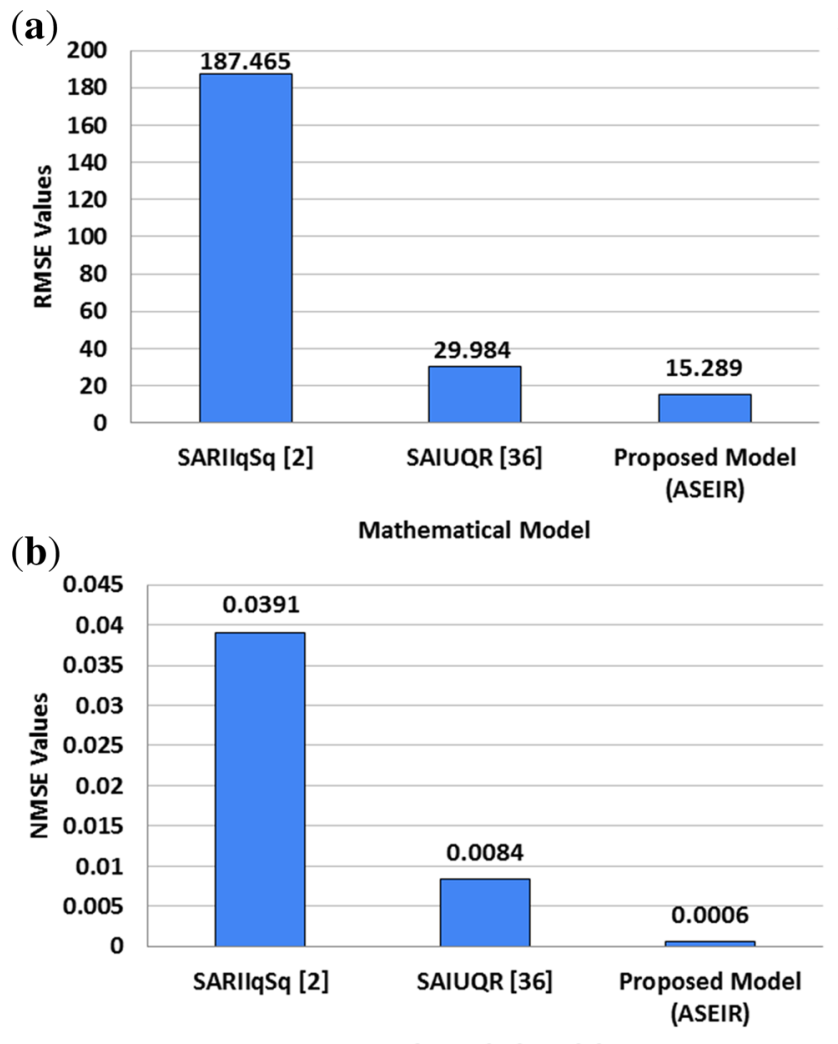

Mathematical Model

Figure 4. Comparative results with state state-of-the-art in terms of (a) RMSE values and (b) NMSE values.

outperforms state-of-the-art models in terms of RMSE as well as the NMSE values.

\section{Conclusion and future extensions}

In this paper, an ASEIR compartmental model for the prediction of the cumulative infectious cases of COVID-19 is formulated. In the mathematical formulation, the following features are included: a new environmental component in the transmission dynamics; variations in the transmission rates for reflecting the impact of coronaviruses; and the evaluation of individual reproduction number to know the mode of transmission of the disease are taken into consideration. Further, the quantification of the severity of the disease is reflected by calculating the basic reproduction number for India with $R_{0}$ equal to 3.57 . The proposed model with the actual data of the cumulative confirmed cases of COVID-19 in India is verified through graphical interpretation, which leads to greater accuracy and minimal error.

No study till now has focused on the concept of humidity or temperature, or any variation of climatic conditions, playing a vital role in determining the spread of this pandemic. Therefore, we consider normal climatic conditions as the basis of the entire calculation. If, in future, some climatic conditions affect the spreading rate of the disease, then we can apply it in the proposed model and evaluate accordingly. In the proposed model, we assume homogenous distribution of population across the countries and their territories. For achieving better accuracy we ignore the assessment of number of deaths and births during our calculation, which can be extended to spatial level where we can divide the territory into smaller segments. The recorded data about the number of COVID-19 patients are volatile. Hence, we can improve our theoretical result and the comparison can become more efficacious once we get accurate data about the infected people on the basis of ample evidence.

\section{References}

[1] Sales J H 2020 Epidemic COVID mathematical model. Int. J. Latest Res. Sci. Technol. 72: 1-5

[2] Sarkar K, Khajanchi S and Nieto J J 2020 Modeling and forecasting the COVID-19 pandemic in India. Chaos Solitons Fractals 139: 110049

[3] Samui P, Mondal J and Khajanchi S 2020 A mathematical model for COVID-19 transmission dynamics with a case study of India. Chaos Solitons Fractals 140: 110173

[4] Rahman S M 2016 Study of infectious diseases by mathematical models: predictions and controls. Ph.D Thesis, University of Western Ontario

[5] Khajanchi S, Bera S and Roy T K 2021 Mathematical analysis of the global dynamics of a HTLV-I infection model, considering the role of cytotoxic T-lymphocytes. Math. Comput. Simul. 180: 354-378

[6] Lizarralde-Bejarano D P, Arboleda-Sanchez S and PuertaYepes M E 2017 Understanding epidemics from mathematical models: details of the 2010 dengue epidemic in Bello (Antioquia, Colombia). Appl. Math. Modell. 43: 566-578

[7] Wu J T, Leung K and Leung G M 2020 Nowcasting and forecasting the potential domestic and international spread of the 2019-nCoV outbreak originating in Wuhan, China: a modelling study. The Lancet 395: 689-697

[8] Tang B, Wang X, Li Q, Bragazzi N L, Tang S, Xiao Y and Wu J 2020 Estimation of the transmission risk of the 2019-nCoV and its implication for public health interventions. J. Clin. Med. 9: 462

[9] Read J M, Bridgen J R, Cummings D A, Ho A and Jewell C P 2020 Novel coronavirus 2019-nCoV: early estimation of epidemiological parameters and epidemic predictions. MedRxiv

[10] Zhu H, Guo Q, Li M, Wang C, Fang Z, Wang P, Tan J, Wu S and Xiao Y 2020 Host and infectivity prediction of Wuhan 2019 novel coronavirus using deep learning algorithm. BioRxiv

[11] Ivorra B, Ferrández M R, Vela-Pérez M and Ramos A M 2020 Mathematical modeling of the spread of the coronavirus disease 2019 (COVID-19) taking into account the undetected infections. The case of China. Commun. Nonlinear Sci. Numer. Simul. 88: 105303 
[12] Inaba H 1990 Threshold and stability results for an agestructured epidemic model. J. Math. Biol. 28: 411-434

[13] Tang B, Xia F, Tang S, Bragazzi N L, Li Q, Sun X, Liang J, Xiao Y and Wu J 2020 The effectiveness of quarantine and isolation determine the trend of the COVID-19 epidemics in the final phase of the current outbreak in China. Int. J. Infect. Dis. 95: 288-293

[14] Yang C and Wang J 2019 A cholera transmission model incorporating the impact of medical resources. Math. Biosci. Eng. 16: 5226-5246

[15] Roberts M G and Tobias M I 2000 Predicting and preventing measles epidemics in New Zealand: application of a mathematical model. Epidemiol. Inf. 124: 279-287

[16] Zhou Y, Ma Z and Brauer F 2004 A discrete epidemic model for SARS transmission and control in China. Math. Comput. Modell. 40: 1491-1506

[17] Gastanaduy P A 2013 Update: severe respiratory illness associated with Middle East Respiratory Syndrome Coronavirus (MERS-CoV)—worldwide, 2012-2013. Morbidity Mortal. Weekly Rep. 62: 480

[18] Frieden T R, Damon I, Bell B P, Kenyon T and Nichol S 2014 Ebola 2014 - new challenges, new global response and responsibility. N. Engl. J. Med. 371: 1177-1180

[19] Chaves L F, Hurtado L A, Rojas M R, Friberg M D, Rodríguez R M and Avila-Aguero M L 2020 COVID-19 basic reproduction number and assessment of initial suppression policies in Costa Rica. Math. Modell. Nat. Phenom. 15: 32

[20] Hamzah F B, Lau C, Nazri H, Ligot D V, Lee G and Tan C L 2020 Coronatracker: worldwide COVID-19 outbreak data analysis and prediction. Bull. World Health Organ.. https:// doi.org/10.2471/BLT.20.255695

[21] Hall R E, Jones C I and Klenow P J 2020 Trading off consumption and COVID-19 deaths. No. W27340, National Bureau of Economic Research

[22] Dong E, Du H and Gardner L 2020 An interactive web-based dashboard to track COVID-19 in real time. Lancet Inf. Dis. 20: $533-534$

[23] MoHFW । Home https://www.mohfw.gov.in/ (last accessed 2020/08/10)
[24] Demographics 2020 Worldometer https://www.world ometers.info/demographics/ (last accessed 2020/08/10)

[25] India population 2020 Live-countrymeters https://country meters.info/en/India (last accessed 2020/08/10)

[26] India population 2020 Worldometer https://www.world ometers.info/world-population/india-population/ (last accessed 2020/08/10)

[27] Firstpost 2020 https://www.firstpost.com/health/coronavirusoutbreak-return-migration-and-covid-19-in-india-data-suggestskerala-tamil-nadu-punjab-up-bihar-may-be-future-red-zonesfor-contagion-risk-8221531.html (last accessed 2020/08/10)

[28] The Indian Express $2020 \mathrm{https} / / /$ indianexpress.com/article/ india/coronavirus-covid-19-tracker-india-news-live-updatescorona-active-cases-deaths-india-lockdown-news-today6418268/ (last accessed 2020/08/10)

[29] Coronavirus Death Rate (COVID-19) 2020 Worldometer, https://www.worldometers.info/coronavirus/coronavirus-deathrate/ (last accessed 2020/08/10)

[30] Who.int I Home https://www.who.int/ (last accessed 2020/08/10)

[31] The Hindu https://www.thehindu.com/news/national/indiarecords-new-daily-high-in-number-of-cases-says-its-recoveryrate-is-40-per-cent/article31636062.ece (last accessed 2020/ $08 / 10$ )

[32] The Times of India https://timesofindia.indiatimes.com/ india/how-asymptomatic-cases-fuelled-spread-of-corona virus/articleshow/75292506.cms (last accessed 2020/08/10)

[33] Hindustan Times https://www.hindustantimes.com/indianews/2-000-under-treatment-3-000-tested-free-for-covid-19under-ayushman-bharat-scheme/story-ROgYn2caqy6gnM MaYDdxQL.html (last accessed 2020/08/10)

[34] Delhi I. Indian Council of Medical Research, New Delhi https://www.icmr.gov.in/ (last accessed 2020/08/10)

[35] Khajanchi S, Sarkar K, Mondal J and Perc M 2020 Dynamics of the COVID-19 pandemic in India. arXiv preprint arXiv: 2005.06286

[36] Khajanchi S and Sarkar K 2020 Forecasting the daily and cumulative number of cases for the COVID-19 pandemic in India. Chaos Interdiscip. J. Nonlinear Sci 30: 071101 